\title{
Article
}

\section{Patients' satisfactions on the waiting period at the emergency units. Comparison study before and during COVID-19 pandemic}

\author{
Abdelhakim Abdelhadi \\ Department of Engineering Management, Prince Sultan University, Riyadh, Saudi Arabia
}

\begin{abstract}
Background: This paper analyzes the patients' experience and satisfaction regarding the waiting period at the emergency unit's hospital before and during the COVID-19 pandemic.

Design and methods: Brainstorming methodology and data analysis from the public domain used on male and female patients in a private hospital in a middle eastern country. The data analyzed seek the patients' level of satisfaction about the waiting period before entering the treatment area, inside the treatment area before the doctor's check, and during the rest period after the doctor's visit. The customer's satisfaction is a significant measure during the COVID-19 pandemic, as it may affect the patient's perspective of the facility. A paired $t$-test at $95 \%$ confidence level was conducted.

Results and Conclusions: The results indicated no difference in satisfaction of the period spent in the emergency room before and during the pandemic.
\end{abstract}

\section{Introduction}

Improving healthcare quality has been a great concern for academics, professionals, and practitioners of healthcare services. Many studies in the literature investigate healthcare service quality and related issues such as patients' dissatisfaction due to the long waiting time. ${ }^{1}$ In this study, literature reviews are related to defining and analyzing the problems related to investigating the improvements in the processing time in the emergency room before and during the COVID 19 pandemic. According to a joint study by the World Health Organization (WHO) ${ }^{2}$ and the World Bank in 2018, low-quality health services hold back progress on improving health in countries at all income levels. WHO also defined the quality of healthcare as:

"The extent to which healthcare services provided to individuals and patient populations improve desired health outcomes Health care must be safe, effective, timely, efficient, equitable, and people-centered." (WHO, 2018).

The meaning of timely in the definition is reducing delays in providing and receiving health care, as described in the study. One of the strategic goals of developing countries is to improve the quality and efficiency of healthcare services. One of the aspects to achieve that goal is by enhancing the patient experience, which is the patient experience center manages at the country's health ministry under this study who initiated the Patient Experience Measurement Program. Due to the current COVID-19 pandemic, healthcare providers should adjust how they define their satisfaction experience. This study will discuss the differences in patients' satisfaction during their visit to an emergency room before and during COVID-19 pandemic. Three aspects of the patient's waiting period in the emergency units are considered as follows:

i) before entering the treatment area

ii) inside the treatment area before the doctor's check, and

iii) the rest in the waiting room after the doctor's visit.

Following is the descriptions and the importance of measuring customers' satisfaction in each stage

\section{Stage one}

The patient satisfaction of the waiting period before entering the treatment area (area 1):

Before entering the treatment area, the waiting period is mainly caused by the increase in the number of patients, lack of healthcare professionals, and the treatment area processes, such as the registration period. The waiting period issue can be addressed by increasing the number of healthcare professionals to cater to the increased number of patients or by hiring part-time professionals to cover the increasing demand. Turning into the electronic filing system for all the citizens and recording all the patient's history and medical records and linking it across the kingdom will help eliminate the waiting time while processing the patients.

\section{Stage two}

The patient satisfaction of the waiting period before the doctor's check (area 2):

The second point is after entering the treatment room and waiting for the doctor's check. The waiting period inside the treatment area before the doctor's examination is another issue. This is mainly attributed to the doctors taking a long time with a single patient, the doctor being late for work, and an increased number of patients compared to the number of available professionals. The issue can be fixed by ensuring that there's no gap between the professional shifts. Besides, increasing the number of professionals attending to the patient and having a consultant for diagnosis and a specific number of specialists related to him perform the recommended action. Nurses can help with minor issues such as dress-

Significance for public health

In any society, patients' experience and satisfaction regarding the waiting period at the emergency unit's hospital is very important. This parameter is particularly significant to measure during the COVID-19 pandemic, because it may affect the patient's perspective of the facility they are visiting. 
ing the injured patients so that the doctor does not take a long time with a single patient.

\section{Stage three}

The patient satisfaction of the rest in the waiting area (area 3):

Rest in the waiting area is another issue caused by the improper design and allocation of the waiting rooms. The waiting rooms' capacity cannot accommodate the number of patients. Also, the improper choice of furniture and the lack of entertainment units. This issue can be adequately addressed by increasing the number of waiting areas around the emergency unit and locate it around the main waiting points. Moreover, to choose suitable furniture that will help the patients and their companions to rest during their waiting period. Providing entertainment activities, such as books or magazines, can also help during the waiting period.

\section{Literature review}

Healthcare professionals should appreciate and implement the importance of patient satisfaction, which is realized by promoting a high standard of care through the quality provision of healthcare services in all hospital departments of a hospital. ${ }^{3}$ Based on the research done by Trout and Hedges, ${ }^{4}$ patient satisfaction is quite an important indicator with regards to the quality of care that is provided by the personnel at any healthcare institution. Boudreaux and $\mathrm{Hea}^{5}$ also established similar findings in a literature review which indicated that the most promising interventions to improve the quality of care in the emergency department include provision of sufficient information using visual media on how the ED operates, enhancing the ED processes by conducting regular performance improvement measures and having routine pieces of training to improve the interpersonal skills of the providers. These findings are supported further by a cross-sectional study to identify the areas associated with significant patient satisfaction in emergency nursing. The results showed that emergency departments should make relevant improvements in educational aspects targeting patients' waiting time and effectively communicating them. ${ }^{6}$

Patient satisfaction is closely tied to hospitals' financial gains as established by a study done by Baugh et al., ${ }^{7}$ which creates an incentive for hospitals to introduce measures that will minimize the waiting times at EDs and provide patient-centered care right after the patient is received at the hospital. Hoffenberg et al. propose utilizing the sharing process to reduce the waiting times. ${ }^{8}$ This is important because patient satisfaction directly affects the patient's choice of physician provider's subsequent choice and the overall perception that the patients have of the healthcare facility. ${ }^{9-}$ 11 Taylor and Benger conducted a systematic review to identify published evidence related to patient satisfaction, specifically in emergency medicine. ${ }^{12}$ This research indicates that the key interventions necessary to improve patient satisfaction have to develop the emergency staff's interpersonal and attitude skills and minimize the waiting time. The research findings were published by Sun et al. ${ }^{13}$ Hospitals should seek care measures at the emergency department that prioritize expedited care to improve patient satisfaction and enhance their willingness to return. In fact, patient satisfaction for the emergency department are improved by ensuring that information is distributed to the patients immediately when they arrive at the emergency department. ${ }^{14}$ In assessing nursing services' role and its impact on patient satisfaction in patients, a previous study established that emergency nursing care is a significant predictor of patient satisfaction. ${ }^{15}$ This indicates the importance of managing patient expectations at the emergency department triage to avoid overcrowding, lengthy waiting times, and uncomfortable waiting room conditions ${ }^{16,17}$ proposes that the emergency team at any given hospital should establish effective communication protocols to enhance the communication with the patient as it is quite crucial towards effective dispensation of nursing care in EDs. Even though the nurse and the patient's expectations may differ in terms of nursing care in the emergency department, nurses should utilize patient satisfaction as a crucial measurable outcome to evaluate what they can improve in nursing practice at the ED. ${ }^{18-20}$

\section{Methods}

The international standards clearly indicate the percentage of satisfaction when a patient waits at the emergency unit before getting medical services. The main aspects that determine the waiting period include the waiting period before entering the treatment area, the waiting period inside the treatment area before the doctor's check, and the rest in the waiting area. These three aspects were measured in a private hospital in the capital of Saudi Arabia in the third quarter of 2019 from the period of July $1^{\text {st }}$ to September $30^{\text {th }}$, as part of a wider survey to establish the levels of patient satisfaction concerning two international standards, namely the Press Ganey (https://www.pressganey.com/). It measures eight major aspects: waiting time upon arrival, nursing, doctors, checkup, pharmacy, family and friends, personal and overall satisfaction. In this paper, some aspects of the appearance regarding the patients' waiting will be discussed. The patients' waiting time is selected to compare before and after the pandemic to improve customers' satisfaction based on the results' outcome.

Data for this project were collected from a public domain repository (https://www.moh.gov.sa/en/Ministry/ OpenData/ Pages/default.aspx) and from (https://velocityglobal. com/blog/abrief-overview-of-health-care-in-the-gcc/), which indicate the average satisfaction percentage of the hospitals and centers in the Golf Countries Counsel countries.

Fifty responses dealt with waiting time before the pandemic, and 50 different responses after the pandemic were selected. Those surveys were taken through phone call surveys and electronic surveys. Questions related to this study were straightforward and consisted of one question related to the customers' satisfaction related to the waiting period in the three stages of their visit to the emergency room. The level of satisfaction was classified into five levels as follows: not at all satisfied, slightly satisfied, moderately satisfied, very satisfied, extremely satisfied.

\section{Results and Discussion}

The satisfaction level is $100 \%$ when the response was extremely satisfied, and $0 \%$ when the customer is not at all satisfied, as shown in Table 1. Sample of the level of satisfaction collected from patients are shown in Table 2.

Table 1. Customer satisfaction rankings.

Level of satisfaction Not at all satisfied
Slightly satisfied Moderately satisfied Very satisfied Extremely satisfied 
Table 2. Customers' level of satisfactions.

\begin{tabular}{|c|c|c|c|c|c|c|}
\hline n & Area 1 & the pan & Amea 3 & Area 1 1 & ig the pand & Area 31 \\
\hline 1 & 75 & 25 & 25 & 25 & 25 & 25 \\
\hline 2 & 50 & 25 & 75 & 100 & 0 & 75 \\
\hline 3 & 75 & 100 & 50 & 25 & 25 & 50 \\
\hline 4 & 0 & 75 & 25 & 25 & 50 & 25 \\
\hline 5 & 75 & 100 & 25 & 75 & 25 & 50 \\
\hline 6 & 75 & 25 & 75 & 100 & 25 & 75 \\
\hline 7 & 25 & 25 & 75 & 75 & 25 & 50 \\
\hline 8 & 0 & 25 & 25 & 75 & 25 & 50 \\
\hline 9 & 50 & 25 & 0 & 100 & 0 & 75 \\
\hline 10 & 50 & 100 & 25 & 0 & 25 & 50 \\
\hline 11 & 25 & 0 & 100 & 100 & 75 & 50 \\
\hline 12 & 50 & 75 & 25 & 75 & 50 & 75 \\
\hline 13 & 50 & 75 & 50 & 20 & 75 & 50 \\
\hline 14 & 25 & 25 & 75 & 75 & 25 & 0 \\
\hline 15 & 25 & 25 & 50 & 100 & 50 & 50 \\
\hline 16 & 50 & 50 & 100 & 50 & 0 & 50 \\
\hline 17 & 75 & 75 & 25 & 0 & 75 & 75 \\
\hline 18 & 75 & 100 & 50 & 22 & 50 & 50 \\
\hline 19 & 75 & 50 & 50 & 100 & 50 & 25 \\
\hline 20 & 50 & 25 & 75 & 50 & 100 & 50 \\
\hline 21 & 25 & 100 & 25 & 50 & 75 & 50 \\
\hline 22 & 25 & 25 & 75 & 75 & 50 & 75 \\
\hline 23 & 75 & 0 & 100 & 100 & 50 & 50 \\
\hline 24 & 25 & 50 & 50 & 50 & 25 & 50 \\
\hline 25 & 25 & 100 & 0 & 50 & 50 & 25 \\
\hline 26 & 100 & 50 & 25 & 75 & 50 & 50 \\
\hline 27 & 50 & 75 & 50 & 75 & 50 & 25 \\
\hline 28 & 25 & 25 & 75 & 25 & 50 & 25 \\
\hline 29 & 25 & 75 & 75 & 50 & 50 & 25 \\
\hline 30 & 0 & 50 & 25 & 75 & 75 & 100 \\
\hline 31 & 25 & 75 & 100 & 100 & 75 & 75 \\
\hline 32 & 75 & 75 & 50 & 25 & 25 & 25 \\
\hline 33 & 50 & 75 & 55 & 25 & 100 & 50 \\
\hline 34 & 75 & 50 & 75 & 25 & 50 & 50 \\
\hline 35 & 25 & 25 & 100 & 75 & 50 & 50 \\
\hline 36 & 50 & 25 & 50 & 25 & 50 & 25 \\
\hline 37 & 0 & 50 & 55 & 50 & 25 & 100 \\
\hline 38 & 75 & 50 & 75 & 0 & 75 & 75 \\
\hline 39 & 50 & 50 & 76 & 25 & 25 & 50 \\
\hline 40 & 100 & 0 & 100 & 50 & 50 & 75 \\
\hline 41 & 100 & 75 & 75 & 100 & 25 & 50 \\
\hline 42 & 50 & 50 & 50 & 25 & 50 & 50 \\
\hline 43 & 50 & 0 & 50 & 25 & 25 & 75 \\
\hline 44 & 75 & 100 & 25 & 50 & 75 & 75 \\
\hline 45 & 50 & 100 & 25 & 75 & 25 & 25 \\
\hline 46 & 75 & 25 & 75 & 75 & 75 & 50 \\
\hline 47 & 75 & 25 & 25 & 25 & 50 & 50 \\
\hline 48 & 50 & 25 & 50 & 25 & 75 & 75 \\
\hline 49 & 25 & 50 & 25 & 25 & 25 & 25 \\
\hline 50 & 50 & 75 & 50 & 75 & 75 & 100 \\
\hline
\end{tabular}


Test of the hypothesis is conducted using $t$-paired test regarding the level of customers' satisfaction during their stay in each emergency area as discussed before
Null hypothesis
$\mathrm{H}_{0}: \mu$ difference $=0$
Alternative hypothesis
$\mathrm{H}_{1}: \mu$ difference $\neq 0$

Where $\mu$ represent the average customers' satisfaction for the population.

According to https:/www.statisticssolutions.com/manovaanalysis-paired-sample-t-test/, the paired sample $t$-test is a statistical procedure used to determine whether the mean difference between two sets of observations is zero. In a paired sample $t$-test, each subject or entity is measured twice, resulting in pairs of observations. In this research, the comparison relates to the customer's satisfaction level in the three mentioned areas: A1 and A2, A1 and $\mathrm{A} 3$, and $\mathrm{A} 2$ and $\mathrm{A} 3$.

The exact time was not considered because we seek satisfaction, and each case has its own duration. The question only relates to the level of satisfaction from the customer's point of view.

Finally, a p-value was used to find a significant difference in the level of customers' satisfaction in each area based on patients' responses to the surveys provided. At $95 \%$ confidence level, if the p-value is less or than or equal to 0.05 , then there are significant differences. Otherwise, no level of significance is presented.

Minitab 19 software is used to run the paired sample $t$-test on the data collected; p-value results are shown in Table 3, while Figure 1 represents a sample of Individual Value Plot of Differences between Area 1 and Area 2 with C.I of $\mathrm{x}$ bar is (-7.86, 1.17). Figure 2 presents comparison of level of satisfactions from customers' point of view between areas 2 and 2 1.

The major conclusion is that there are no significant differences between their answers, but the satisfaction level after the pandemic is lower.

This research used data before the COVID-19 pandemic as part of one of the strategic goals to achieve the 2030 vision of The Kingdom of Saudi Arabia required by the Ministry of Health is to improve the quality and efficiency of the services provided by the ministry. There are many ways to improve patient satisfaction in the emergency departments, including increasing the number of beds to accommodate more patients in line with increasing the

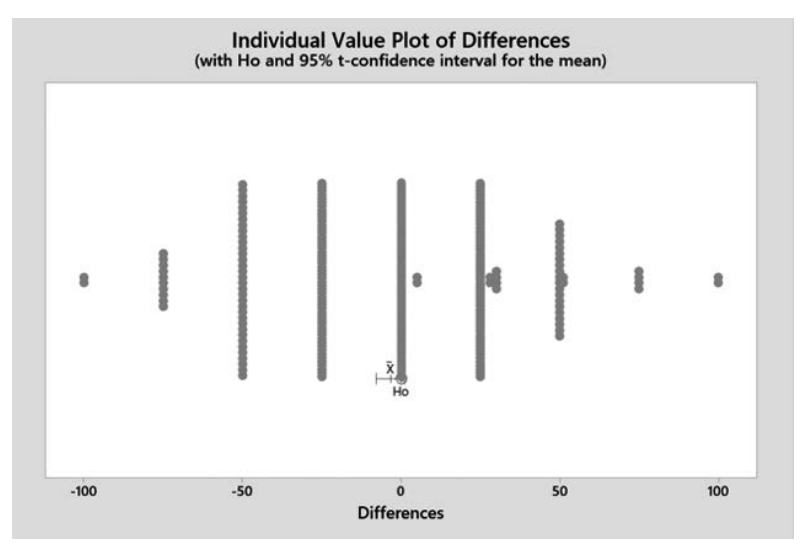

Figure 1. Individual value plot of differences between Area 1 and Area 2 before the pandemic. number of doctors. At the first encounter with patients, the triage physician should facilitate patients' existence from the emergency department. Moreover, he considered seeing patients quickly. Lastly, educating the patients about the emergency department's work dynamics through education screens and pamphlets. The purpose of the new survey is to check if there are any changes in patients' perceptions to the emergency department or if there are some changes in medical staff toward the emergency patients due to the COVID-19 pandemic.

\section{Conclusions}

Health is one of the major focus areas of the Vision 2030 strategic plan with one of the major aspects of this goal requiring patient experience improvement. Patient satisfaction is one of the key performance measures that can be applied to evaluate healthcare quality. In this paper, the patient's waiting period in the emergency units was considered to give insights into patients' quality of care before and during the COVID-19 pandemic. The research analysis indicates no difference in customers' satisfaction of the main aspects that determine the waiting period through the surveys. This includes the waiting period before entering the treatment area, the waiting period inside the treatment area before the doctor's check, and the rest in the waiting area. The survey results indicate no differences in satisfaction between the three sections of the emergency units before and after the pandemic. This raises the reason behind this conclusion, which should lead to doing another research concentrating on this outcome.

However, healthcare facilities should develop requisite meas-

Table 3. p-value of the $t$-paired test.

\begin{tabular}{lc} 
Test & p-value \\
﹎_difference: mean of (Area 1 - Area 2) & 0.721 \\
\hline __difference: mean of (Area 1 - Area 3) & 0.420 \\
﹎difference: mean of (Area 2 - Area 3) & 0.753 \\
\hline ﹎difference: mean of (Area 1_1 - Area 3_1) & 0.718 \\
__difference: mean of (Area 2_1 - Area 3_1) & 0.159 \\
\hline __difference: mean of (Area 2_1 - Area 1_1) & 0.196
\end{tabular}

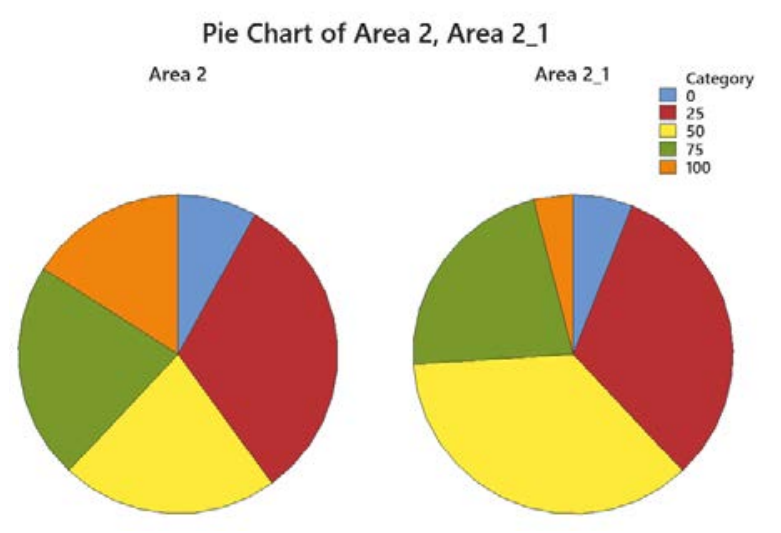

Figure 2. Comparison of level of satisfactions from customers' point of view between areas 2 and 2 _1. 
ures of assessing these aspects to determine the interventions that should be introduced to improve the quality of care. At the first waiting point after check-in, delays are mainly caused by the lack of enough healthcare professionals and poor organizational care systems. At the second point after entering the treatment room, the average waiting period depends on the number of patients assigned per doctor. The third point is the resting area. The research indicates that a below-average patient satisfaction rate is mainly attributed to poor organization and physical resources distribution around the main waiting points.

Patient satisfaction is an integral indicator of the personnel's quality of care at any healthcare institution. To improve the quality of care in emergency departments across the country, the research proposes that the emergency department units' required interventions should be patient-centered to improve the overall health outcomes.

Correspondence: Abdelhakim Abdelhadi, Department of Engineering Management, Prince Sultan University, Riyadh, Saudi Arabia. E-mail: tungrah@yahoo.com

Key words: Patient satisfaction; emergency room; COVID-19.

Acknowledgments: The author would like to thank the Prince Sultan University for financial support to produce this research.

Conflict of Interest: The author declares that he has no known competing financial interests or personal relationships that could have influenced the work reported in this paper.

Ethics approval and consent to participate: Not required as the study is based on secondary data, not containing personal and/or sensitive personal data.

Availability of data and materials: Data for this project were collected from public domain repositories.

Received for publication:19 September 2020.

Accepted for publication: 22 February 2021.

oCopyright: the Author(s), 2021

Licensee PAGEPress, Italy

Journal of Public Health Research 2021;10:1956

doi:10.4081/jphr.2021.1956

This work is licensed under a Creative Commons Attribution NonCommercial 4.0 License (CC BY-NC 4.0).

\section{References}

1. Abdelhadi A. Investigating emergency room service quality using lean manufacturing. Int $\mathrm{J}$ Health Care Quality Ass 2015;28:510-9.

2. Alodan A, Alalshaikh G, AlqasabHi, et al. Studying the effi- ciency of waiting time in outpatient pharmacy. MethodsX 2020;7:100913.

3. Naidu A. Factors affecting patient satisfaction and healthcare quality. Int J Health Care Quality Ass 2009;22:366-81.

4. Trout A, Magnusson AR, Hedges JR. Patient satisfaction investigations and the emergency department: what does the literature say? Acad Emerg Med 2000;7:695-709.

5. Boudreaux ED, Hea EL. Patient satisfaction in the emergency department: a review of the literature and implications for practice. J Emerg Med 2004;26:13-26.

6. Messina G, Vencia F, Mecheroni S, et al. Factors affecting patient satisfaction with emergency department care: an Italian rural hospital. Glob J Health Sci 2015;7:30.

7. Baugh CW, Venkatesh AK, Bohan J S. Emergency department observation units: a clinical and financial benefit for hospitals. Health Care Manage Rev 2011;36:28-37.

8. Hoffenberg S, Hill MB, Houry D. Does sharing process differences reduce patient length of stay in the emergency department? Ann Emerg Med 2001;38:533-40.

9. Thompson DA, Yarnold PR, Williams DR, Adams SL. Effects of actual waiting time perceived waiting time, information delivery, and expressive quality on patient satisfaction in the emergency department. Ann Emerg Med 1996;28:657-65.

10. Khankeh HR, Khorasani-Zavareh D, Azizi-Naghdloo F, et al. Triage effect on the wait time of receiving treatment services and patients satisfaction in the emergency department: Example from Iran. Iran J Nurs Midwifery Res 2013;18:79.

11. Dansky KH, Miles J. Patient satisfaction with ambulatory healthcare services: waiting time and filling time. J Healthcare Manage 1997;42:165.

12. Taylor C, Benger JR. Patient satisfaction in emergency medicine. Emerg Med J 2004;21:528-32.

13. Sun BC, Adams J, Orav EJ, et al. Determinants of patient satisfaction and willingness to return with emergency care. Ann Emerg Med 2000;35:426-34.

14. Krishel S, Baraff LJ. Effect of emergency department information on patient satisfaction. Ann Emerg Med 1993;22:568-72.

15. ALmutairi MN, El.Mahalli AA. Burnout and coping methods among emergency medical services professionals. J Multidiscip Healthc 2020;13:271-9.

16. Shah S, Patel A, Rumoro DP, et al. Managing patient expectations at emergency department triage. Patient Exper J 2015;2:31-44.

17. McCarthy DM, Ellison EP, Venkatesh AK, et al. Emergency department team communication with the patient: the patient's perspective. J Emerg Med 2013;45:262-70.

18. Blank FS, Tobin J, Jaouen M, et al. A comparison of patient and nurse expectations regarding nursing care in the emergency department. J Emerg Nurs 2014;40:317-22.

19. Raposo ML, Alves HM, Duarte PA. Dimensions of service quality and satisfaction in healthcare: a patient's satisfaction index. Serv Bus 2009;3:85-100.

20. Alumran A, Alkhaldi O, Aldroorah Z, et al. Utilization of an electronic triage system by emergency department nurses. J Multidiscip Healthc 2020;13:339-44. 